\title{
THE FUNCTIONS OF A COMPLEX VARIABLE DEFINED BY AN ORDINARY DIFFERENTIAL EQUATION OF THE FIRST
}

\author{
ORDER AND THE FIRST DEGREE*
}

BY

\section{JOSEPH SLEPIAN}

Beginning with Cauchy's $\dagger$ fundamental theorem in 1820, much has been done toward answering the two fundamental questions: Do there exist solutions of a given differential equation or system of equations, which satisfy given initial conditions, and how far are such solutions determined by these initial conditions?

The case in which the coefficients of the system are analytic throughout the domain of the independent variable was in the main disposed of by Cauchy and his contemporaries, but the cases in which the coefficients present singularities, has continued to occupy the attention of mathematicians up to the present time. Almost all of these researches, however, are concerned with the existence and nature of a solution only in a certain restricted domain for the independent variables.

In the present paper we propose to study the ordinary equation of the first order and first degree, $d y / d x=P(x, y) / Q(x, y)$, where $P(x, y)$ and $Q(x, y)$ are polynomials in the two complex variables $x$ and $y$, for all values of $x$ and $y$. The first part of the paper is given over to a statement of the well-known existence theorems required later. The functions defined by such an equation will present no other singularities than branch points or poles, except possibly at a finite number of points which may be determined algebraically, and these solutions are in general infinitely many valued functions over the $x$-plane.

These functions have been largely investigated by Boutroux $\ddagger$ who studies the manner of interchange of their branches and the grouping of their branch

* Presented to the Society, April 26, 1913.

$\dagger$ Picard, Traité d'analyse, vol. 2, chap. 11.

$\ddagger$ Rendiconti del Circolo Matematico di Palermo, vol. 24 (1907), pp. 209-222. Journal de mathématiques, ser. 6, vol. 6 (1910), pp. 137-199. Leçons sur les fonctions définies par les équations différentielles du premier ordre. GauthierVillars, Paris, 1908. 
points in the neighborhood of the fixed singularities, and the manner of growth ("croissance") of single branches of these functions over extended regions in the $x$-plane.

In this paper we shall not make a study of the individual integrals of an equation, but rather of the "im grossen" properties of the totality of all the integrals of the equation. We shall be guided by the well-known facts in the case of real variables, due largely to Poincaré.* In this case the solutions may be represented by a family of curves in the $x, y$-plane; there are a finite number of singular points through which more than one curve may pass, and there are certain closed limit curves or cycles about which other curves wind spirally.

The second part of the present paper shows that the closed cycle has a close analogy in the complex case; here every solution of the equation has limit solutions which, with respect to this integral, play a part analogous to that of the closed cycle in the real case. These limit solutions are in general infinite in number, whereas in the real case the limiting closed cycles are finite in number. However, by applying a sieve-like process due to Birkhoff, $\dagger$ we are led to certain cyclic systems of limit solutions which have notable properties. There is in general only one such system, which probably coincides in general with the totality of solutions, but which does not always do so.

The last three parts of this paper deal with the interrelation of the fixed singularities, limit solutions, and cyclic systems of limit solutions. In particular, it is shown that, if all the fixed singularities of the equation are of the simplest type, any two integrals pass through a common fixed singularity or have a limit solution in common. The case when algebraic solutions exist is especially considered.

I wish here to express my thanks to Professor G. D. Birkhoff for his kind assistance and many suggestions in preparing this paper.

\section{Preliminary theorems}

The principal existence theorem in the theory of differential equations is that of Cauchy.

Theorem of Cauchy. Given the differential equation, $d y / d x=f(x, y)$. If $f(x, y)$ is analytic in the region, $\left|x-x_{0}\right| \leqq r,\left|y-y_{0}\right| \leqq \rho$ then there exists one and only one function of $x, y(x)$, which satisfies the given equation, which at the point $x_{0}$ takes on the value $y_{0}$, and which is analytic within a circle about $x_{0}$, whose radius is not less than

$$
r\left(1-e^{-\rho /(2 M r)}\right)
$$

*Journal de mathématiques, ser. 3, vol. 7 (1881), p. 375 and vol. 8 (1882), p. 251.

†Bulletin de la Société mathématique de France, vol. 40 (1912), pp. 305-323. 
where $M$ is the maximum absolute value of $f(x, y)$ in the region

$$
\left|x-x_{0}\right| \leqq r, \quad\left|y-y_{0}\right| \leqq \rho .
$$

Throughout this paper, as in this theorem, by the term "a circle about a point," we shall mean a circle whose center is at that point.

Picard* has further proved that if a function which satisfies the differential equation can be extended analytically along a path $L$ to $x_{0}$ (but perhaps not beyond), and if this function when taken along $L$ to $x_{0}$ approaches the value $y_{0}$, then this function must be identical with the function $y(x)$ of the theorem. This result shows that the function under these conditions can be extended past the point $x_{0}$.

For the "im grossen" theory of the first order differential equation,

$$
d y / d x=P(x, y) / Q(x, y)
$$

where $P$ and $Q$ are polynomials without common factors, we need to consider the point at infinity in both the $x$ - and the $y$-planes.

If we make the transformation, $\bar{y}=1 / y$ we obtain a new equation of the same form, $d \bar{y} / d x=P_{2}(x, \bar{y}) / Q_{2}(x, \bar{y})$, whose integrals are the reciprocals of the integrals of the given equation. If $P_{2}(x, y) / Q_{2}(x, y)$ is analytic at $x=x_{0}$, $y=0$, then by the theorem of Cauchy there is a function $\bar{y}(x)$, which satisfies the equation $d \bar{y} / d x=P_{2}(x, \bar{y}) / Q_{2}(x, \bar{y})$ and which is analytic and vanishes at $x_{0}$. If $\bar{y}(x)$ does not vanish identically, then there is one, and only one, function of $x, y(x)$ which satisfies the equation $d y / d x=P(x, y) / Q(x, y)$ and which at the point $x_{0}$ has a pole. If the function $\bar{y}(x)$ vanishes identically then the equation $d y / d x=P(x, y) / Q(x, y)$ has no integral which at $x_{0}$ becomes infinite. In this case, to secure uniformity of statement in the theorems of this paper, we shall say that the equation admits the ideal integral $y=\infty$.

To treat the case where $x$ is infinite, we make the transformation, $\bar{x}=1 / x$, obtaining the equation, $d y / d \bar{x}=P_{1}(\bar{x}, y) / Q_{1}(\bar{x}, y)$; and, to treat the case where both $x$ and $y$ are infinite, we make the transformation, $\bar{x}=1 / x, \bar{y}=1 / y$, obtaining $d \bar{y} / d \bar{x}=P_{3}(\bar{x}, \bar{y}) / Q_{3}(\bar{x}, \bar{y})$. We obtain results for these cases entirely similar to the one stated for the case where $y$ becomes infinite.

Thus, if the point at infinity is treated after the manner indicated, it is found to play a part no different from a finite point in the $x$ - or $y$-plane. Throughout this paper, even if no special mention of the fact is made, the possibility of a variable becoming infinite is not to be excluded.

We have next to consider the points at which $P(x, y) / Q(x, y)$ (or the corresponding quotient $P_{i} / Q_{i}$ ) fails to be analytic. These points have been divided into two categories, the movable singularities, and the fixed or essen-

\footnotetext{
* Picard, Traité d'analyse, vol. 2, chap. 11.
} 
tial singularities. The first category is made up of these cases:

(a): $x_{0}, y_{0}$ finite, $\quad Q\left(x_{0}, y_{0}\right)=0, \quad Q\left(x_{0}, y\right) \neq 0, \quad P\left(x_{0}, y_{0}\right) \neq 0$;

(b): $\quad x_{0}$ infinite, $\quad Q_{1}\left(0, y_{0}\right)=0, \quad Q_{1}(0, y) \neq 0, \quad P_{1}\left(0, y_{0}\right) \neq 0$;

(c): $\quad y_{0}$ infinite, $\quad Q_{2}\left(x_{0}, 0\right)=0, \quad Q_{2}\left(x_{0}, y\right) \neq 0, \quad P_{2}\left(x_{0}, 0\right) \neq 0$;

$(d): \quad x_{0}, y_{0}$ infinite, $\quad Q_{3}(0,0)=0, \quad Q_{3}(0, y) \neq 0, \quad P_{3}(0,0) \neq 0$.

The following theorem is characteristic of these cases.

Theorem. If $Q\left(x_{0}, y_{0}\right)=0, Q\left(x_{0}, y\right) \neq 0$, and $P\left(x_{0}, y_{0}\right) \neq 0$, then there is one and only one function of $x, y(x)$ which satisfies the differential equation, $d y / d x=P(x, y) / Q(x, y)$, takes on the value $y_{0}$ at $x_{0}$ and has at $x_{0} a$ simple branch point.

Here also the solution is unique in the sense defined above.

The other category of singularities of $P(x, y) / Q(x, y)$, the fixed singularities, we find it convenient to further subdivide into two classes, denoting them respectively as fixed singularities of the first kind, and of the second kind. The fixed singularities of the first kind are the points $x_{0}, y_{0}$ as follows:

(a): $\quad x_{0}, y_{0}$ finite, $\quad P\left(x_{0}, y_{0}\right)=0, \quad Q\left(x_{0}, y_{0}\right)=0, \quad Q\left(x_{0}, y\right) \neq 0$;

(b): $\quad x_{0}$ infinite, $\quad P_{1}\left(0, y_{0}\right)=0, \quad Q_{1}\left(0, y_{0}\right)=0, \quad Q_{1}(0, y) \neq 0$;

(c): $\quad y_{0}$ infinite, $\quad P_{2}\left(x_{0}, 0\right)=0, \quad Q_{2}\left(x_{0}, 0\right)=0, \quad Q_{2}\left(x_{0}, y\right) \neq 0$;

$(d): \quad x_{0}, y_{0}$ infinite, $\quad P_{3}(0,0)=0, \quad Q_{3}(0,0)=0, \quad Q_{3}(0, y) \neq 0$.

A singularity of this kind has evidently two coördinates, $x_{0}, y_{0}$, so that at $x_{0}$ only those integrals which there take on the value $y_{0}$ fail to come under the preceding theorems.

The fixed singularities of the second kind include the only remaining singularities, i. e., the cases where $Q\left(x_{0}, y\right) \equiv 0, Q_{1}(0, y) \equiv 0, Q_{2}\left(x_{0}, y\right) \equiv 0$, or $Q_{3}(0, y) \equiv 0$, if any of these cases occur. A singularity of this kind has evidently only one coördinate $x_{0}$; at such a point $x_{0}$, the preceding theorems fail to apply, irrespective of the initial value of $y_{0}$. It is evident there are only a finite number of fixed singularities of either the first or second kind.

By the aid of the preceding theorems, and a further theorem first proved by Painlevé,* we can state that a function of $x$ which satisfies the equation $d y / d x=P(x, y) / Q(x, y)$ can be extended over the whole $x$-plane, and, ; except at the $x$-coördinates of the fixed singularities, the only singularities of the function will be poles and branch points. In general such functions will be infinitely many valued.

Let $f\left(x, x_{0}, y_{0}\right)$ denote that integral of the equation, which at the point $x_{0}$

* See Boutroux, Leçons sur les fonctions définies par les équations différentielles, p. 15. 
has the value $y_{0}$. It is shown by various writers, that $f\left(x, \bar{x}_{0}, \bar{y}_{0}\right)$ which depends obviously upon $\bar{x}_{0}, \bar{y}_{0}$ as well as upon $x$, is algebroid in its three arguments in the neighborhood of $x_{0}, x_{0}, y_{0}$, provided that $x_{0}, y_{0}$, or $x_{0}$, are not the coördinates of a fixed singularity of the first kind, or second kind, respectively.

Furthermore, $f\left(x, \bar{x}_{0}, \bar{y}_{0}\right)$ is algebroid in its three arguments in the neighborhood of $\left(x_{1}, x_{0}, y_{0}\right)$, where $x_{1}$ and $x_{0}$ are the extremities of a path $L$ which passes through the $x$-coördinate of no fixed singularity, and along which $f\left(x, x_{0}, y_{0}\right)$ has no branch point except possibly at $x_{1}$. For the purposes of this paper, it is necessary to note further that if at the point $x_{1}$, for fixed $\left(x_{0}, y_{0}\right), f\left(x, x_{0}, y_{0}\right)$ has a pole or branch point of order $k$, then there exists a neighborhood of $x_{1}$, and one of $\left(x_{0}, y_{0}\right)$, such that if $\left(\bar{x}_{0}, \bar{y}_{0}\right)$ is in the latter neighborhood, then $f\left(x, \bar{x}_{0}, \bar{y}_{0}\right)$ for fixed $\left(\bar{x}_{0}, \bar{y}_{0}\right)$ will have poles or branch points in the former neighborhood, the sum of whose orders is $k$. This extension follows immediately from the proof of the preceding statement.

\section{Limit SOLUTIONS AND CYCLIC SYSTEMS}

The integrals of a differential equation, $d y / d x=P(x, y) / Q(x, y)$ are in general infinitely many valued functions over the $x$-plane. The limiting determinations of the infinitely many determinations of an integral of the differential equation form what we shall call the limit solutions of that integral. Let us give an exact definition:* At a point $X$, which is not the $x$-coördinate of a fixed singularity, let $Y_{1}, Y_{2}, Y_{3}, \cdots$ be the determinations of an integral $Y(x)$ counted as distinct if obtained by analytic extension along essentially distinct paths. Then if $Z$ be any limiting value of $Y_{1}, Y_{2}, Y_{3}, \cdots$ the integral $Z(x)$ defined by $f(x, X, Z)$ is a limit solution of $Y(x)$.

Although the definition requires only that infinitely many determinations of $Y(x)$ are near a particular determination, $Z$, of $Z(x)$ only at $X$, it readily follows from the algebroid character of $f(x, \bar{x}, \bar{y})$ in its arguments that in the neighborhood of a determination of $Z(x)$ at any $x$ not the $x$-coördinate of a fixed singularity, there are infinitely many determinations of $Y(x)$.

For, the determination $Z$ at the point $X$ can be carried into any other determination at any point $x$ not the $x$-coördinate of a fixed singularity, over a pa th $L$, which avoids all the fixed singularities and branch points of $Z(x)$. Since determinations of $Y(x)$ in any neighborhood of $Z$ exist at $X$, determinations of $Y(x)$ differing from $Z(x)$ by as little as we please, may be found at any point $x$ on $L$.

The proof of the following obvious theorem is omitted.

*Cf. Boutroux, Annales de éécole normalesupérieure, ser. 3, vol. 27 (1905), p. 448. C o m p tes R e n d us, vol. 138, p. 850-3. Leçons sur les fonctions définies par les équations différentielles du premier ordre, pp. 25-30. 
Theorem I. A limit solution of a limit solution of an integral of the differential equation $d y / d x=P(x, y) / Q(x, y)$ is again a limit solution of that integral.

In general, every integral will have infinitely many limit solutions, each limit solution will have infinitely many limit solutions, etc. In thus forming limit solutions of limit solutions the question naturally arises as to whether there exists a set of integrals such that the limit solutions of any integral of the set are the same set of integrals. An integral with a finite number of values obviously is a special case of such a set. I shall now proceed to show that there exists always a set of integrals having this property.

Let us think of the $y$-plane as stereographically projected upon the $y$-sphere in the usual manner. Take any integral $Y(x)$ of the equation

$$
d y / d x=P(x, y) / Q(x, y),
$$

and consider its various determinations at a point $X$, not the $x$-coördinate of a fixed singularity, and suppose these determinations to be marked upon the $y$-sphere. Suppose likewise that all the determinations at the point $X$ of all the limit solutions of $Y(x)$ are marked upon the $y$-sphere. The two sets of marked points together form a closed set, of course.

Now, suppose the sphere to be divided up into a finite number of simple sub-regions. A particular limit solution of $Y(x)$ may have determinations lying in every one of the sub-regions of the sphere, or it may fail to have any determination in some of the sub-regions. For our present purposes, it is most convenient to have each sub-region include its boundary, so that a boundary point will lie in two or more regions.

Let $Y_{1}(x)$ be either $Y(x)$ or a limit solution of $Y(x)$ whose determinations lie in a least number of these sub-regions; that is, any other limit solution of $Y(x)$ must have determinations entering into at least as many sub-regions of the $y$-sphere as do the determinations of $Y_{1}(x)$. Having chosen $Y_{1}(x)$, let us reject from further consideration all those sub-regions of the sphere which contain no determination of $Y_{1}(x)$. All the determinations of the limit solutions of $Y_{1}(x)$ will evidently lie only in the sub-regions which have been retained, and will be among the points already marked.

Now, divide up the retained sub-regions into smaller subregions, still following the convention that the boundary of a region is part of that region. Determinations of $Y_{1}(x)$ or of any particular one of its limit solutions may lie in every one of these smaller subregions, or only in some of them. Let $Y_{2}(x)$ be one of these integrals, whose determinations enter into a least number of the smaller sub-regions. Now reject from further consideration all the sub-regions which do not contain determinations of $Y_{2}(x)$.

This process of sub-division and selection of $Y_{1}(x), Y_{2}(x) \cdots$ may be 
continued indefinitely. If the largest dimension of the largest sub-region in the successive divisions approaches zero, a minimal set of points, $L$, which will be retained through this process, will have the following property.

Theorem II. An integral of the equation which at $x=X$ has one of its determinations belonging to a minimal set $L$ must have all its determinations at $x=X$ belonging to the set.

Proof. First let us remark that a necessary and sufficient condition that a point $Z$ be retained in the process of division and rejection, is that the point $Z$ be a limit point of the determinations of every one of the integrals $Y_{1}(x)$, $Y_{2}(x), \cdots$. The sufficiency is obvious, for since the sub-regions are always supposed to retain their boundaries, after the $k$ th division, the limit points of the determinations of $Y_{k}(x)$ must be retained.

The necessity is shown thus: Suppose that $Z$ were not a limit point of the determinations of $Y_{k}(x)$. Then we could surround $Z$ by a region $T$ on the sphere, in which there would be no determinations of $Y_{k}(x)$ and therefore also no determinations of $Y_{k+1}(x), Y_{k+2}(x), \cdots$.

At some division, say the $l$ th, $l>k, Z$ would be an interior point of a sub-region lying wholly within $T$, or would be a boundary point of subregions all of which would lie wholly within $T$. Now since $Y_{l}(x)$ has no determinations in $T, Z$ would necessarily be rejected with the sub-regions of which it is an interior or boundary point.

Thus we see that if an integral has one of its determinations belonging to $L$ that integral must be a limit solution of $Y_{1}(x), Y_{2}(x), \cdots$. But in that case any other determination of the integral is a limit point of determinations of every one of the integrals, $Y_{1}(x), Y_{2}(x), \cdots$, and that is sufficient that this determination belong to $L$.

Theorem III. If an integral has determinations at $X$ belonging to a minimal set $L$, then any limit solution of this integral has at $X$ determinations belonging to the set $L$.

For the given integral must be a limit solution of all the integrals $Y_{1}(x)$, $Y_{2}(x), \ldots$. Hence so too must be its limit solution. But this suffices to make its limit solution have determinations among the set $L$.

TheOREM IV. Any integral which at $X$ has determinations belonging to a minimal set $L$, is a limit solution of any other integral which at $X$ has determinations belonging to the set $L$.

For suppose that $Z_{1}(x)$ and $Z_{2}(x)$ are integrals which at $X$, respectively, have determinations belonging to the set $L$, and that $Z_{1}(x)$ is not a limit solution of $Z_{2}(x)$. Let $Z$ be any determination of $Z_{1}(x)$. Then at some stage in the process of division and rejection by which the set $L$ was obtained, say the $k$ th stage, $Z$ must lie in a sub-region which contains no determination of $Z_{2}(x)$ or must be a boundary point of regions which contain 
no determinations of $Z_{2}(x)$. But since $Z_{2}(x)$ is a limit solution of $Y_{k}(x)$, its determinations do not enter into more sub-regions of the $k$ th division than do the determinations of $Y_{k}(x)$. Also since $Z$ is retained throughout the whole process of division and rejection the determinations of $Y_{k}(x)$ do enter the sub-regions of which $Z$ is an interior or boundary point, but the determinations of $Z_{2}(x)$ do not. Hence, the determinations of $Z_{2}(x)$ enter into a fewer number of sub-regions than do those of $Y_{k}(x)$ and therefore $Y_{k}(x)$ is not a limit solution of $Y_{k-1}(x)$ which enters irto a least number of subregions of the $k$ th division. Thus, we get a contradiction.

Definition. A set of integrals such that any limit solution of any member of the set is again a member of the set, and such that any member of the set is a limit solution of any other member of the set, shall be called a cyclic system.

Theorems III and IV show the existence of cyclic systems, and it is clear from the method of constructing the set $L$ that every integral of the equation $d y / d x=P(x, y) / Q(x, y)$ has at least one cyclic system of limit solutions.

The integrals of a cyclic system are essentially alike over any region not including a fixed singularity. For, taking a branch of one integral of a cyclic system in the neighborhood of a point $x$ not the $x$-coördinate of a fixed singularity, then, from the definition, branches of any other integral of the system may be found which differ indefinitely little from the branch of the chosen integral in the neighborhood of $x$. Moreover this neighborhood may be taken indefinitely large so long as it includes no fixed singularity.

Theorem V. No two different cyclic systems of the same equation can have an integral in common.

For, from the definition, a cyclic system may be generated from a single one of its members by forming all the limit solutions of that member.

In the next section of this paper, we shall see that, because of this theorem, in a large number of cases there cannot be more than one cyclic system for a single equation. It appears probable that, in general, in these cases, the cyclic system will consist of the totality of integrals of the equation. In these cases every integral is a limit solution of any one integral, so that the determination of a single integral in its entirety is equivalent to determining every integral of the equation.

Examples.

$$
\frac{d y}{d x}=y \text {. }
$$

The general integral is $y=C e^{x}$. All the integrals are single valued functions, and therefore each individual integral is a cyclic system by itself. Here, then, we have infinitely many distinct cyclic systems.

$$
\frac{d y}{d x}=\frac{\lambda y}{x} \text {. }
$$


The general integral is

$$
y=C x^{\lambda}
$$

If $\lambda$ is real and irrational, two distinct integrals, $y=C_{1} x^{\lambda}, y=C_{2} x^{\lambda}$ will be limit solutions of each other if and only if, $C_{1} / C_{2}$ has absolute value equal to unity. In this case if $C$ is any constant, the family of integrals $y=e^{i \phi} C x^{\lambda}$ obtained by giving $\phi$ all real values will form a cyclic system. Here, too, we have infinitely many cyclic systems.

The general integral is

$$
\frac{d y}{d x}=\frac{y^{2}}{x}
$$

$$
y=-\frac{1}{\log x+C}
$$

The integral $y \equiv 0$ is a limit solution of every integral of the equation. Hence here there is only one cyclic system, and this consists of the integral $y=0$.

$$
\frac{d y}{d x}=\frac{\lambda_{1}}{2 \pi i} \frac{1}{x-a_{1}}+\frac{\lambda_{2}}{2 \pi i} \frac{1}{x-a_{2}}+\frac{\mu_{1} i}{2 \pi i} \frac{1}{x-a_{3}}+\frac{\mu_{2} i}{2 \pi i} \frac{1}{x-a_{4}} .
$$

Here we shall assume that $a_{1}, a_{2}, a_{3}, a_{4}$, are distinct, that $\lambda_{1}$ and $\lambda_{2}$ are reals relatively incommensurable to each other, and that $\mu_{1}$ and $\mu_{2}$ are reals relatively incommensurable to each other.

The general integral is

$$
\begin{aligned}
y=\frac{\lambda_{1}}{2 \pi i} \log \left(x-a_{1}\right)+\frac{\lambda_{2}}{2 \pi i} \log \left(x-a_{2}\right)+\frac{\mu_{1} i}{2 \pi i} \log ( & \left.x-a_{3}\right) \\
& +\frac{\mu_{2} i}{2 \pi i} \log \left(x-a_{4}\right)+C .
\end{aligned}
$$

If $y_{0}$ is a determination of any integral at a point $x_{0}$, then all the other determinations of that integral are given by

$$
y_{0}+\left(m_{1} \lambda_{1}+m_{2} \lambda_{2}\right)+i\left(n_{1} \mu_{1}+n_{2} \mu_{2}\right)
$$

where $m_{1}, m_{2}, n_{1}, n_{2}$, independently take on all integral values positive and negative.

But, inasmuch as $\lambda_{1}$ and $\lambda_{2}$ are relatively incommensurable, we may choose $m_{1}, m_{2}$, so as to make $m_{1} \lambda_{1}+m_{2} \lambda_{2}$ indefinitely near to any preassigned real number $a$. Similarly we may choose $n_{1}, n_{2}$ so as to make $n_{1} \mu_{1}+n_{2} \mu_{2}$ indefinitely near to any preassigned real number $b$.

Hence, at the point $x$ considered, determinations of the integral exist, which come indefinitely near to any previously chosen complex number, $Z$, whatever. Hence, since the integral considered was any integral, every 
integral of the equation has as limit solutions all the integrals of the equation.

Hence, for this equation, the totality of all the integrals forms a cyclic system.

\section{Fixed singularities and CYCtic systems}

The simplest type of fixed singularity, i. e., one where the curves

$$
P(x, y)=0 \text { and } Q(x, y)=0
$$

have a simple intersection, has been much studied. Briot and Bouquet,* in a classic memoir on this subject, proved the following facts. Let $P(x, y)$ and $Q(x, y)$ when expanded into power series about $\bar{x}, \bar{y}$, begin respectively with the terms $a(x-\bar{x})+b(y-\bar{y})+\cdots, c(x-\bar{x})+d(y-\bar{y})+\cdots$. Then since the intersection is simple, we have $a d-b c \neq 0$. Hence neither root of the equation for $\lambda, \lambda^{2}-(a+d) \lambda+a d-b c=0$, can be zero. Let the two roots of this equation be $\lambda_{1}$ and $\lambda_{2}$. When these two roots are distinct, the integrals of the equation in the neighborhood of $\bar{x}, \bar{y}$ are given by $g(x, y)^{\lambda_{1}} / h(x, y)^{\lambda_{2}}=C$, where $C$ is an arbitrary constant, and $g(x, y)$ and $h(x, y)$ are functions of $x, y$, and analytic and vanishing at $\bar{x}, \bar{y}$ with non-vanishing jacobian. The functions of $x$ defined by $g(x, y)=0$ and $h(x, y)=0$ will be integrals of the differential equation.

THEOREM VI. $\dagger$ If $\lambda_{1}$ and $\lambda_{2}$ are distinct and their ratio is not real, then the two integrals of the differential equation given by $g(x, y)=0$ and $h(x, y)=0$ are limit solutions of all the other integrals of the equation which come within a certain neighborhood of $\bar{x}, \bar{y}$.

Proof. Since the jacobian of $g(x, y)$ and $h(x, y)$ does not vanish at $\bar{x}, \bar{y}$, we may map the neighborhood of $\bar{x}, \bar{y}$ into a neighborhood of 0,0 , by the transformation $\xi=g(x, y), \eta=h(x, y)$. This transformation will be one to one and analytic. Then the "curves" $g(x, y)^{\lambda_{1}} / h(x, y)^{\lambda_{2}}=C$ will go over into the curves $\xi^{\lambda_{1}} / \eta^{\lambda_{2}}=C$; or $\eta=C^{-1 / \lambda_{1}} \xi^{\lambda_{1} / \lambda_{2}}$. Suppose that the coefficient of the pure imaginary part of the ratio $\lambda_{1} / \lambda_{2}$ is positive. If then on any of the "curves" $\eta=C^{-1 / \lambda_{2}} \xi^{\lambda_{1} / \lambda_{2}}$ we let $\xi$ circle the origin in the positive sense, staying at a constant distance from it, $\eta$ will approach the value zero. Hence $\eta \equiv 0$ will be a limit solution of all the curves of the family $\eta=C^{-1 / \lambda_{2}} \xi^{\lambda_{1} / \lambda_{2}}$ and therefore the curve $g(x, y)=0$ will be a limit solution of the family $g(x, y)^{\lambda_{1}} / h(x, y)^{\lambda_{2}}=C$. If the coefficient of the pure imaginary in the ratio $\lambda_{1} / \lambda_{2}$ is negative, we derive the same conclusion by letting $\xi$ circle the origin in the negative sense. In a similar fashion we

*Journal de l'école polytechnique, vol. 21 (1856), pp. 161-198.

† Cf. Boutroux, Leçons sur les fonctions définies par les équations différentielles du premier ordre, p. 116. Rendiconti del Circolo matematico di Palermo, vol. 24 (1907), pp. 209-222. 
may show that the curve $h(x, y)=0$ is a limit solution of every curve of the family.

CoRollary. The integrals of only one cyclic system can pass in the neighborhood of a fixed singularity $\bar{x}, \bar{y}$, of the type in Theorem VI, unless the integrals defined by $g(x, y)=0$, and $h(x, y)=0$ are finitely many-valued functions of $x$, in which case each respectively will form a distinct cyclic system.

For by Theorem VI, all cyclic systems which pass in the neighborhood of $\bar{x}, \bar{y}$, must have as members $g(x, y)=0$, or $h(x, y)=0$, and therefore by Theorem $\mathrm{V}$, there cannot be more than two distinct cyclic systems in the neighborhood of $\bar{x}, \bar{y}$. Now suppose $g(x, y)=0$ yields an infinitely manyvalued function, and belongs to a cyclic system. Then there must be infinitely many other members of the system which pass arbitrarily near $\bar{x}, \bar{y}$. But by Theorem VI these must have $h(x, y)=0$ as a limit solution. Hence there cannot be more than one cyclic system of integrals in the neighborhood of $\bar{x}, \bar{y}$. Similarly if $h(x, y)=0$ is infinitely many valued there cannot be more than one cyclic system in the neighborhood of $\bar{x}, \bar{y}$.

If $g(x, y)=0$ and $h(x, y)=0$ are respectively finitely many valued, then each will obviously be a cyclic system.

Example.

$$
\frac{d y}{d x}=-\frac{\lambda(x-y)+\mu(x+y)}{\lambda(x-y)-\mu(x+y)} .
$$

The conditions of Theorem VI at the point $(0,0)$ are satisfied if $\lambda / \mu$ is not real.

The general integral is $(y+x)^{\lambda} /(y-x)^{\mu}=C$. In this case

$$
g(x, y)=y+x \text {, and } h(x, y)=y-x .
$$

Here $g(x, y)=0$ and $h(x, y)=0$ are respectively cyclic systems.

\section{A fundamental theorem}

The remainder of this paper is confined to the equation whose fixed singularities are all of the first kind.

If the equation $d y / d x=P(x, y) / Q(x, y)$ is to have no finite singularity of the second kind, it is evidently necessary and sufficient that $Q(x, y)$ shall have no factor of the form $x-x_{0}$. If there is to be no singularity of the second kind at infinity, it is necessary and sufficient that the degree of $Q(x, y)$ in $x$, shall exceed that of $P(x, y)$ in $x$ by at least two.

An integral of such an equation which is finitely many valued must be algebraic. For it is everywhere algebroid except possibly at the finite number of fixed singularities; at these however, because they are of the first kind, the integral must remain finite or become infinite. Hence by generalizations of 
the theorem on removable singularities, the integral must be algebroid at the fixed singularities, and, being everywhere algebroid, must be algebraic.

We come now to this

Fundamental theorem, Part I. If $Y_{1}(X)$ and $Y_{2}(X)$ are two integrals of an equation, $d y / d x=P(x, y) / Q(x, y)$, all of whose fixed singularities are of the first kind, and if $Y_{1}(x)$ has no algebraic limit solution which differs by a constant from an algebraic limit solution of $Y_{2}(x)$, then either $Y_{1}(x)$ and $Y_{2}(x)$ have a limit solution in common, or $Y_{1}(x)$ and $Y_{2}(x)$ each pass through a common fixed singularity, $i$. e., when $x$ tends along some path in the $x$-plane to the point $\bar{x}$, both $Y_{1}(x)$ and $Y_{2}(x)$ will approach the common value $\bar{y}$, where $\bar{x}, \bar{y}$, are the coördinates of a fixed singularity.

PART II. If $Y_{1}(x)$ has algebraic limit solutions which differ only by constants from limit solutions of $Y_{2}(x)$, and if none of these limit solutions are themselves constants, then $Y_{1}(x)$ and $Y_{2}(x)$ come arbitrarily near a common fixed singularity.

Proof of Part $I$. We shall suppose that $Y_{1}(x)$ and $Y_{2}(x)$ have no limit solution in common and show they must then pass through a common fixed singularity.

With this hypothesis we have the following lemmas:

LеммA I. Let the $x$-coördinates of the fixed singularities and the point infinity in the $x$-plane be surrounded by arbitrary circles, $C$, respectively. Then there exists a positive number $D$, and a positive integer $k$, such that in any circle in the $x$-plane of radius $D$, not overlapping the $C$ circles, there are not more than $k$ branch points and zeros of the function $Y_{1}^{\prime}(x)-Y_{2}^{\prime}(x) .^{*}$

Suppose the lemma were not true. Take a sequence of number pairs, $D^{\prime}, k^{\prime} ; D^{\prime \prime}, k^{\prime \prime} ; D^{\prime \prime \prime}, k^{\prime \prime \prime}$; etc., such that the $D^{\prime}$ s are approaching zero, and the $k$ 's are becoming infinite. Then for any pair $D^{(i)}, k^{(i)}$ it must be possible to find $x^{(i)}, y_{1}^{(i)}, y_{2}^{(i)}$, such that $\hat{f}_{1}^{\prime}\left(x, x^{(i)}, y_{1}^{(i)}\right)-f_{2}^{\prime}\left(x, x^{(i)}, y_{2}^{(i)}\right)$ has more than $k^{(i)}$ branch points and zeros inside a circle about $x^{(i)}$ of radius $D^{(i)}$, where $f_{1}\left(x, x^{(i)}, y^{(i)}\right)$, and $f_{2}\left(x, x^{(i)}, y_{2}^{(i)}\right)$ are respectively branches of $Y_{1}(x)$ and $Y_{2}(x)$. Let $X$ be a limit point of the $x^{(i)}$ 's. Choose a sequence of $x^{(i)}$ 's, which approach $X$, and let $Y_{1}$ be a limiting value of the corresponding

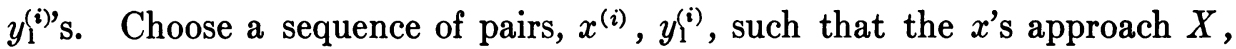
and the $y$ 's approach $Y_{1}$. Let $Y_{2}$ be a limit point of the corresponding set $y_{2}^{(i)}$.

Now $f_{1}^{\prime}\left(x, X, Y_{1}\right)-f_{2}^{\prime}\left(x, X, Y_{2}\right)$ has at $X$ a branch point of finite order, say the $l$ th, and a zero of finite order, say the $m$ th, provided that

$$
f_{1}^{\prime}\left(x, X, Y_{1}\right)-f_{2}^{\prime}\left(x, X, Y_{2}\right),
$$

does not vanish identically. But if $f_{1}^{\prime}\left(x, X, Y_{1}\right)-f_{2}^{\prime}\left(x, X, Y_{2}\right) \equiv 0$,

\footnotetext{
* Accents here and later in the paper indicate differentiation.
} 
then integrating, we have $f_{1}\left(x, X, Y_{1}\right)-f_{2}\left(x, X, Y_{2}\right) \equiv C$, a constant. This constant cannot be zero since by hypothesis $Y_{1}(x)$ and $Y_{2}(x)$ have no limit solution in common. Further, we must have

$$
\frac{P\left(x, f_{1}\right)}{Q\left(x, f_{1}\right)}=\frac{P\left(x, f_{2}\right)}{Q\left(x, f_{2}\right)}=\frac{P\left(x, f_{1}-c\right)}{Q\left(x, f_{1}-c\right)}=\frac{P\left(x, f_{2}+c\right)}{Q\left(x, f_{2}+c\right)} .
$$

Hence $f_{1}\left(x, X, Y_{1}\right)$ and $f\left(x, X, Y_{2}\right)$ would be respectively algebraic, and differ from each other only by a constant. But this case is ruled out in the hypothesis of the theorem.

Then by the algebroid character (in the extended sense noted) of $f(x, X, Y)$ in its three arguments, we may find a neighborhood of $X$, and neighborhoods of $X, Y_{1}$, and $X, Y_{2}$, such that if $x^{(i)}, y_{1}^{(i)}$, and $x^{(i)}, y_{2}^{(i)}$ be respectively in the latter two neighborhoods, then $f_{1}^{\prime}\left(x, x^{(i)}, y^{(i)}\right)-f_{2}^{\prime}\left(x, x^{(i)}, y_{2}^{(i)}\right)$ as a function of $x$ will have branch points and zeros in the former neighborhood, the sum of whose orders does not exceed $l+m$. But this contradicts the defining property of the point $X, Y_{1}, Y_{2}$.

Lemma 2. Let the $x$-coördinates of the fixed singularities, and the point infinity in the $x$-plane, be surrounded by arbitrary circles $C$. Then for any positive number $E$, there exists a positive number $M$ such that if a point $x_{1}$ is outside the circles $C$, and if in a circle of radius $E$ about this point $x_{1}$ (this circle not overlapping the $C$ circles), $Y_{1}^{\prime}(x)-Y_{2}^{\prime}(x)$ has no zero, we have

$$
\left|Y_{1}^{\prime}\left(x_{1}\right)-Y_{2}^{\prime}\left(x_{1}\right)\right|>M \text {. }
$$

Suppose the lemma were not true. Then, having chosen $E$ arbitrarily, choose a sequence of $M^{\prime}$ 's approaching zero: $M^{\prime}, M^{\prime \prime}$, etc. Then for any $M^{(i)}$ we can find values, $x^{(i)}, y_{1}^{(i)}, y_{2}^{(i)}$, such that in a circle of radius $E$ about the point $x$ and not overlapping the $C$ circles, there is no zero of the function $f_{1}^{\prime}\left(x, x^{(i)}, y^{(i)}\right)-f_{2}^{\prime}\left(x, x^{(i)}, y_{2}^{(i)}\right)$, but nevertheless such that

$$
\left|f_{1}^{\prime}\left(x, x^{(i)}, y_{1}^{(i)}\right)-f_{2}^{\prime}\left(x, x^{(i)}, y_{2}^{(i)}\right)\right|<M^{(i)} \text {. }
$$

Let $X$ be a limiting value of the $x^{(i)}$ 's. Choose a sequence of $x^{(i)}$ 's which approach $X$. Let $Y_{1}$ be a limit value of the corresponding $y^{(i)}$ 's. Choose a sequence of pairs, $x^{(i)}, y^{(i)}$, such that the $x$ 's approach $X$ and the $y_{1}$ 's approach $Y_{1}$. Let $Y_{2}$ be a limit point of the corresponding $y^{(i)}$ 's. Now, just as in proof of the preceding lemma, $f_{1}^{\prime}\left(x, X, Y_{1}\right)-f_{2}^{\prime}\left(x, X, Y_{2}\right)$ cannot vanish identically. Suppose that $f_{1}^{\prime}\left(x, X, Y_{1}\right)-f_{2}^{\prime}\left(x, X, Y_{2}\right)$ has a zero at $X$. Then there is a neighborhood of $X$, and this neighborhood may be taken arbitrarily small, and a corresponding one of $X, Y_{1}, Y_{2}$, such that if $x^{(i)}$, $y_{1}^{(i)}, y_{2}^{(i)}$, be in the latter neighborhood then $f_{1}^{\prime}\left(x, x^{(i)}, y_{1}^{(i)}\right)-f_{2}^{\prime}\left(x, x^{(i)}, y_{2}^{(i)}\right)$ has a zero in the former neighborhood. Suppose that

$$
f_{1}^{\prime}\left(x, X, Y_{1}\right)-f_{2}^{\prime}\left(x, Y, X_{2}\right)
$$


is not zero at the point $X$. Then we can find a number $m$, and a neighborhood of $X$ and a neighborhood of $\left(X, Y_{1}, Y_{2}\right)$ such that if $x^{(i)}, y_{1}^{(i)}, y_{2}^{(i)}$, is in the latter neighborhood, then throughout the former neighborhood $f_{1}^{\prime}\left(x, x^{(i)}, y_{1}^{(i)}\right)-f_{2}^{\prime}\left(x, x^{(i)}, y_{2}^{(i)}\right)$ in absolute value exceeds $m$. Thus in either case we are led to a contradiction.

We are now ready to prove the first part of the fundamental theorem.

Let $Y_{1}(x)$ and $Y_{2}(x)$ be the two integrals having no limit solutions in common. At any point $x$ in the $x$-plane, not the $x$-coördinate of a fixed singularity, form the function $w(x)=Y_{1}(x)-Y_{2}(x)$ taking any branch of each integral respectively.

Suppose $w(x)$ is laid out on its Riemann surface. The only singularities of $w(x)$ on this surface will be branch points and poles, and singularities in the various sheets at the $x$-coördinates of the fixed singularities. The totality of these singularities is denumerable.*

Consider the lines of flow of $w(x)$ on the Riemann surface, i. e., those curves along which arc $w$ is constant, and consider the analytical extension of such a curve in the direction of decreasing $|w|$. This extension can terminate in these two ways; (1), the curve may approach a point at which $|w|$ approaches zero; (2) the curve may approach a singularity. If a curve exists which terminates in the manner (1), then the main theorem is established. For $w(x)=Y_{1}(x)-Y_{2}(x)$ can equal zero only by $Y_{1}(x)$ and $Y_{2}(x)$ going through a common fixed singularity. Suppose now there is no curve, arc $w=$ constant, which terminates in the manner (1). Then at the singularities of $w(x), w \neq 0$, and the values of arc $w$ at these singularities is denumerable. But the totality of values of arc $w$ on the surface is nondenumerable. Hence we may find curves, arc $w=$ constant which do not terminate in the manner (2). Since by hypothesis there are no curves which terminate in the manner (1), these curves cannot terminate at all.

Consider such a non-terminating line of flow. Clearly we may surround the point at infinity, and the $x$-coördinates of the fixed singularities, in the $x$-plane by circles $C$, such that this line of flow does not continually remain within one of these circles.

Suppose $|w|$ approaches 0 along the line of flow. Then by reasoning similar to that used in proving the two lemmas, it may be shown that $Y_{1}(X)$ and $Y_{2}(X)$ would have a limit solution in common. Hence along the line of flow we have $|w|>d>0$.

Let $\rho$ be less than the number $D$ of Lemma 1 . Clearly $\rho$ may be taken so small, that we may cut an infinite number of distinct segments of the line of flow by circles of radius $\rho$, having their centers on the line of flow, and not

* Cf. Poincaré, Rendiconti del Circolo matematico di Palermo, vol. 2 (1888), pp. 197-200. 
overlapping the $C$ circles. Let such a circle of radius $\rho$ have its center at a point $O$ (see Fig. 1), cutting the segment $L M$ of the line of flow of $|w|$.

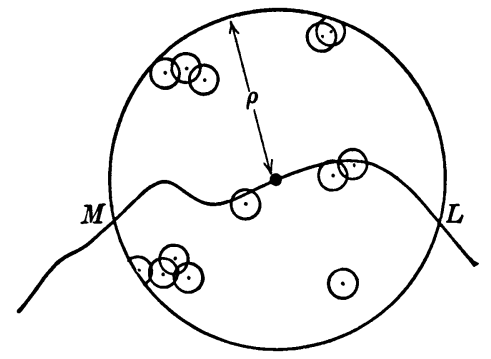

FIg. 1

Then by Lemma I, there are not more than $K$ branch points or poles of $Y_{1}^{\prime}(X)-Y_{2}^{\prime}(X)$ within this circle.

Choose for the number $E$ of Lemma 2, $\rho / 2 k$. Surround the branch points and poles of $Y_{1}(x)-Y_{2}(x)$ which lie within the circle of radius $\rho$ by circles of radius $\rho / 2 k$. Then segments of $L M$ will be outside these circles of radius $\rho / 2 k$, and the combined length of these segments will be not less than $\rho / 2$. On these segments by Lemma 2, $\left|Y_{1}^{\prime}(x)-Y_{2}^{\prime}(x)\right|>M>0$. Hence along these segments,

$$
\left|\frac{d \log w}{d x}\right|=\left|\frac{Y_{1}^{\prime}(x)-Y_{2}^{\prime}(x)}{Y_{1}(x)-Y_{2}(x)}\right|>\frac{M}{d} .
$$

But along the line of flow,

$$
\left|\frac{d \log w}{d x}\right|=-\frac{d \log |w|}{d s}>\frac{M}{d} .
$$

Hence along the segment $L M, \log |w|$ diminishes by more than $M \rho / d$.

Hence since an infinite number of segments such as $L M$ may be found along the line of flow of $|w|, \log |w|$ decreases indefinitely along the line of flow. But this contradicts the hypothesis that $d>0$.

Proof of Part II. Suppose that a pair of limit solutions $Z_{1}(x), Z_{2}(x)$ of $Y_{1}(x)$ and $Y_{2}(x)$ respectively satisfy the relation $Z_{1}^{\prime}(x)-Z_{2}^{\prime}(x) \equiv 0$ so that $Z_{1}$ and $Z_{2}$ are algebraic. Then

$$
f_{1}^{\prime}\left(x, X, Y_{1}\right)-f_{2}^{\prime}\left(x, X, Y_{2}\right)
$$

may vanish identically. But since $Z_{1}(x)$ is not a constant it will certainly have a pole for some value of $x$. But by the relation $Z_{1}^{\prime}(x)-Z_{2}^{\prime}(x) \equiv 0$ it follows that $Z_{2}(x)$ also has a pole for this value of $x$. Hence $Z_{1}(x)$ and $Z_{2}(x)$ pass through a common fixed singularity. But now, if a limit solution of an integral passes arbitrarily near to a fixed singularity, then that 
integral also must pass arbitrarily near to that fixed singularity. Hence $Y_{1}(x)$ and $Y_{2}(x)$ must come respectively arbitrarily near to the common fixed singularity of $Z_{1}(x)$ and $Z_{2}(x)$, which proves the second part of the theorem.

If a pair of limit solutions $Z_{1}(x), Z_{2}(x)$ reduce to constants, it is no longer true as in the preceding paragraph, that they will pass through a common fixed singularity.

\section{The solutions and the fiXed singularities. Algebraic solutions}

THEOREM VII. If an integral stay uniformly away from a fixed singularity, then so do all its limit solutions.

By staying uniformly away, we mean that if $(\bar{x}, \bar{y})$ is the fixed singularity, and $Y(x)$ the integral, then $x$ and $y$ do not come within arbitrarily prescribed neighborhoods of $(\bar{x}, \bar{y})$. With this explanation the theorem becomes obvious.

THEOREM VIII. If all the fixed singularities of an equation are of the simplest type, and if for all of them the ratio of $\lambda_{1}$ to $\lambda_{2}$ is not real (see section 3), then there cannot be more than one cyclic system of integrals of the equation, unless all the cyclic systems reduce to algebraic integrals.

For suppose there were two systems, one not reducing to an algebraic integral. Then consider any pair of integrals, taken one from each cyclic system respectively. By Theorems III and V, we see that they cannot have a limit solution in common. They must therefore go through a common fixed singularity. But all the fixed singularities are of the type for which the corollary to Theorem VI applies, and since one of the cyclic systems does not reduce to a finitely valued integral, it must be the only cyclic system in the neighborhood of that fixed singularity.

Theorem IX. If all the fixed singularities of an equation are of the simplest type, and if for all of them the ratio of $\lambda_{1}$ to $\lambda_{1}$ is not real (see section 3), then there cannot be more than $n$ algebraic integrals not reducing to constants, where

$$
\frac{n(n-1)}{2}+k=m, \quad 0<k \leqq n,
$$

where $n$ and $k$ are integers, and $m$ is the total number of fixed singularities.

For these integrals must pass in pairs through different fixed singularities.

We now consider the following important question: Are there any integrals of an equation, which stay uniformly away from all the fixed singularities of the equation? Obviously, an integral $y=$ constant, must always pass through fixed singularities.

THEOREM $\mathrm{X}$. If there is any integral of the equation which stays uniformly 
away from all the fixed singularities of the equation,* then there is a cyclic system all of whose integrals stay uniformly away from the fixed singularities, and there is no other cyclic system of the equation. The integrals of this cyclic system are limit solutions of every integral of the equation.

For let us form a limiting cyclic system (see Theorem III) from the given integral. This will stay away uniformly from the fixed singularities by Theorem VII. As we have noted, such a cyclic system cannot reduce to a constant. There can be no other cyclic system, for an integral of another system would have to have a fixed singularity in common with every integral of the first cyclic system. Also, since any integral of this system cannot have a fixed singularity in common with any of the integrals of the equation, it must be a limit solution of every integral of the equation.

An algebraic integral is a cyclic system of integrals in itself. Hence we have these corollaries:

CoRollary I. If there is an algebraic integral of the equation which passes through a fixed singularity, then there are no integrals of the equation which stay uniformly away from the fixed singularities.

CoROLlaRY II. If there is an integral $y=$ constant, then there are no integrals of the equation which stay uniformly away from the fixed singularities.

CoRollary III. If there are two algebraic integrals of the equation, then there are no integrals of the equation which stay uniformly away from the fixed singularities.

If the degree of $P(x, y)$ in $y$ does not exceed the degree of $Q(x, y)$ in $y$ by as much as 2 , then the transformation, $\bar{y}=1 / y$, will carry the given equation into one which admits the integral, $\bar{y} \equiv 0$. Hence we get the corollary:

Corollary IV. If the degree of $P(x, y)$ in $y$ alone, does not exceed the degree of $Q(x, y)$ in $y$ alone by as much as 2 , then there are no integrals of the equation which stay uniformly away from the fixed singularities.

Theorem XI. If the curves $P(x, y)=0$, and $Q(x, y)=0$, have only finite intersections in the projective sense, and if all these intersections are simple, then any algebraic integral must pass through a fixed singularity.

Let the integral be given by the algebraic equation, $F(x, y)=0$. Let the total degrees of $P(x, y), Q(x, y), F(x, y)$, be respectively, $p, q, f$. Let the degrees of $P(x, y), Q(x, y), F(x, y), F_{x}(x, y), F_{y}(x, y)$ in $x$ alone respectively by $p_{1}, q_{1}, f_{1}, f_{1 x}, f_{1 y}$, and in $y$ alone respectively by $p_{2}, q_{2}$, $f_{2}, f_{2 x}, f_{2 y}$.

The case where $p_{2}-q_{2}<2$ has been disposed of by Corollary 4 so that we have the relations

$$
p_{2} \geqq q_{2}+2,
$$

* The restriction is still kept that all the fixed singularities of the equation are of the first kind, see section 5 . 


$$
q_{1} \geqq p_{1}+2 \text {. }
$$

Now since $F(x, y)$ represents an integral of the equation

$$
P F_{y}+Q F_{x} \equiv G F,
$$

where $G(x, y)$ is a polynomial whose total degree we shall denote by $g$, and whose degree in $x$ alone and in $y$ alone respectively by $g_{1}$ and $g_{2}$. We have then these relations,

$$
\begin{gathered}
f_{1 x}=f_{1}-1, \\
f_{2 x} \leqq f_{2}, \\
f_{1 y} \leqq f_{1}, \\
f_{2 y}=f_{2}-1 .
\end{gathered}
$$

From (2), (3), (4), (6), we have

$$
q_{1}+f_{1}-1 \geqq g_{1}+f_{1} \text { whence } q_{1}-1 \geqq g_{1} \text {, }
$$

and from (1), (3), (5), (7),

$$
p_{2}+f_{2}-1 \geqq g_{2}+f_{2} \text { whence } p_{2}-1 \geqq g_{2} \text {. }
$$

Suppose that $p \geqq q$. Then from (3)

$$
p+f-1 \geqq g+f, \text { or } p-1 \geqq g \text {. }
$$

Now if the algebraic integral $F(x, y)=0$ passes through no fixed singularity, the curve $F(x, y)=0$, will not pass through any of the intersections of the curves $P(x, y)=0$, and $Q(x, y)=0$. Hence from (3), $G(x, y)$ must pass through all the intersections of $P(x, y)=0$ and $Q(x, y)=0$, and since these are simple, they will be $p q$ in number. Hence $G(x, y)=0$ must meet $Q(x, y)=0$ in $p q$ points, and by (10) we have $p q>g q$. Hence $G(x, y)$ does contain $Q(x, y)$ as a factor, or $G(x, y) \equiv L(x, y) Q(x, y)$. But this contradicts (8).

Similarly we are led to a contradiction if $q>p$.

Corollary. If the curves $P(x, y)=0$ and $Q(x, y)=0$ have only simple finite intersections in the projective sense, and if there is a single algebraic integral, then there are no integrals of the equation which stay uniformly away from the fixed singularities. 